\title{
Research on Some Conclusions of the Distance Spectrum of a Graph
}

\author{
Zhihui Ma \\ College of Sciences, Shihezi University, Shihezi, XinJiang 832003, China \\ mazhihui@shzu.edu.cn
}

Keywords: Distance spectrum; Spectral radius; Some graph

\begin{abstract}
This paper studies some graph distance spectrum, first introduces the basic conce pts that used in this article, and related terms and the main results obtained mark in this $p$ aper. At the same time the paper depicts the matching number is $\beta$, order number is $n$ that has minimum distance spectral radius of graph in all connected graph. The article finally $\mathrm{c}$ alculated the distance spectrum of $\mathrm{G} \odot \mathrm{K} 2$ and $G_{1} \square G_{2} \square G_{3}$.
\end{abstract}

\section{Introduction}

Spectral graph theory is one of the important research fields of graph theory and combinatorial theory, it widely apply on quantum chemistry, physics, computer science, communication networks and information science and other fields. It mainly refers to the adjacency spectrum and the Laplace spectrum, in addition to the distance spectrum et al. Study on spectral graph theory is by using the algebraic theory of mature combined with the structure, properties and combinatorial theory of graph and matrix theory (especially the non-negative matrix theory and combinatorial matrix theory) to study various structural properties of matrix of graph spectra and the spectra and graphs, diagrams other invariants (such as chromatic number, connectivity) between the contact, also will be the conclusion of graph theory used for the study of matrix theory. The both development of a graph spectrum theory and matrix theory is complement each other.

In recent years, the distance spectrum has become a research hot of mapping theory. In this paper, we obtained the pole figure minimum distance spectral radius of the given independence number, independence number are given pole figure to the minimum distance spectral radius of a graph, and the chromatic number of a given pole figure to the minimum distance spectral radius of a graph, and through the comparison of the distance spectrum and adjacent spectral properties and conclusions, we can get the general research approach of distance spectrum summary.

\section{The basic concept and definition}

In this paper we consider the graph $\mathrm{G}$ is a simple connected $\mathrm{n}$ order graphs. The vertex set is $V(G)=\left\{v_{1}, v_{2}, \ldots, v_{n}\right\} . d_{G}\left(v_{i}, v_{j}\right)$ is distance of the vertex $v_{i}$ and $v_{j}$, refers to the shortest length of connecting $v_{i}$ and $v_{j}$ in G.

Definition 1: the distance matrix $D$ of figure $G$ defined as $D=D(G)=\left(d_{i j}\right)_{n^{*} n}$, among $\operatorname{dij}=d_{G}\left(v_{i}\right.$, $\mathrm{v}_{\mathrm{j}}$ ).

Definition 2: all the eigenvalues of $D(G)$ and its eigenvalue multiplicity constitutes a map distance of $\mathrm{G}$ spectrum.

Definition 3: The maximum distance eigenvalues of graph $G$ known as the distance spectral radius, denoted as $\Lambda 1(\mathrm{G})$.

Definition 4: Figure $G=(V, E)$ is called the critical factor, refers to any vertex $v \in V(G), G-v$ has 1-factor.

Definition 5: We call a points set $S \subseteq V$ that match G-S, refer to graph Hs (by each branch of G-S shrinkage a vertex and delete all S edges) containing a saturated $\mathrm{S}$ matching.

Definition 6: We give two graph $\mathrm{G}$ and $\mathrm{H}$, corona of graph $\mathrm{G}$ and $\mathrm{H}$ denote as $\mathrm{G} \odot \mathrm{H}$, among:

$V(G \odot H)=V(G) \cup \bigcup_{i \in V(G)} V\left(H_{i}\right), E(G \odot H)=E(G) \cup \bigcup_{i \in V(G)} E\left(H_{i}\right) \cup\left\{\left(i, u_{i}\right): i \in V(G), u_{i} \in V\left(H_{i}\right)\right\}$. 
Definition 7: We give two graph $\mathrm{G}$ and $\mathrm{H}$, Cartesian product of graph $\mathrm{G}$ and $\mathrm{H}$ define as $V(G \square H)=V(G) \times V(H), \quad E(G \square H)=\{(u, v)(x, y) \mid u=x, v y \in E(H)$, or $u x \in E(G), v=y\}$

Among them, $u, x \in V(G), v, y \in V(H)$

Definition 8: Let the vertex set of graph $G$ is $V(G)=\{v 1, v 2, \ldots, v n\}$, The distance matrix is $D$. VI distance is defined as $D_{i}=\sum_{j=1}^{n} d_{i j}, i=1,2, \ldots, n$.

Define 9: Let distance degree sequence of $G$ is $\{D 1, D 2, \ldots, D n\}$. For all $I$, if $D i=k$, then $G$ is called the $\mathrm{k}$ - distance regular.

Define 10: set $A=\left(a_{i j}\right) \in C^{m \times n}, B=\left(b_{i j}\right) \in C^{r \times s}$, Kronecker Product of A and B is defined as:

$$
A \otimes B=\left(\begin{array}{cccc}
a_{11} B & a_{12} B & \ldots & a_{1 n} B \\
a_{21} B & a_{22} B & \ldots & a_{2 n} B \\
\ldots & \ldots & \ldots & \ldots \\
a_{m 1} B & a_{m 2} B & \ldots & a_{m n} B
\end{array}\right)
$$

\section{The distance spectrum of some graph}

Distance spectrum of $\mathbf{G} \odot \mathbf{K} 2$. Lemma 1: Let $\mathrm{D}$ be a distance matrix of connectivity distance regular graph $G$, then $D$ is irreducible, and there is a polynomial $P(x)$ that makes $P(D)=J$.

$$
P(x)=p \times \frac{\left(x-\lambda_{2}\right)\left(x-\lambda_{3}\right) \ldots\left(x-\lambda_{g}\right)}{\left(k-\lambda_{2}\right)\left(k-\lambda_{3}\right) \ldots\left(k-\lambda_{g}\right)}
$$

$\mathrm{K}=\mathrm{D} 1$, is D's biggest single characteristic root, $\lambda 1, \lambda 2, \ldots, \lambda 9$ is other different characteristic root.

Lemma 2: Set that adjacency matrix of a graph $G$ is $A$, graph spectra of $G$ is $\operatorname{spec}(G)=\{\lambda 1, \lambda$ $2, \ldots, \lambda \mathrm{p}\}$, so:

$$
\operatorname{det} A=\prod_{i=1}^{p} \lambda_{i}
$$

In addition, for any non-zero polynomial $\mathrm{P}(\mathrm{x})$, if $\lambda$ is characteristic value $\mathrm{A}$, then $\mathrm{P}(\lambda)$ is $\mathrm{P}(\mathrm{A})$ feature values. Therefore:

$$
\operatorname{det} P(A)=\prod_{i=1}^{p} P\left(\lambda_{i}\right)
$$

Lemma 3: Set $A=\left(\begin{array}{cc}A_{0} & A_{1} \\ A_{1} & A_{0}\end{array}\right)$ is $2 x 2$ block symmetric matrix. The characteristics value of $A$ is eigenvalues of $\mathrm{A} 0+\mathrm{A} 1$ and $\mathrm{A} 0-\mathrm{A} 1$.

Lemma 4: Set $A=\left(\begin{array}{cc}A & B \\ C & D\end{array}\right)$ is matrix, and $A$ is a non-singular matrix, then:

If $\mathrm{A}$ and $\mathrm{C}$ can be exchanged, it is

$$
\left|\begin{array}{ll}
A & B \\
C & D
\end{array}\right|=|A|\left|D-C A^{-1} B\right|
$$

$$
\left|\begin{array}{ll}
A & B \\
C & D
\end{array}\right|=|A D-C B|
$$

Theorem 5: let $\mathrm{G}$ be a connected distance regular graph, distance regular degree is $\mathrm{k}$, the corresponding distance matrix is $\mathrm{D}$, spec $(\mathrm{G}) 1=\mathrm{k}=\{\mu 1=\mathrm{k}, \mu 2, \ldots \mu \mathrm{n}\}$, and the distance between the feature graph $\mathrm{G} \odot \mathrm{K} 2$ value does not contain distance feature value of $\mathrm{G}$, then 


$$
\begin{aligned}
& \operatorname{spec}_{D}\left(G \quad K_{2}\right)=\left\{-1.5+2 n+1.5 k+0.5 \sqrt{9-24 n-6 k+24 n^{2}+24 n k+9 k^{2}},\right. \\
& -1.5+2 n+1.5 k-0.5 \sqrt{9-24 n-6 k+24 n^{2}+24 n k+9 k^{2}}, 1.5\left(\mu_{i}-1\right)+0.5 \sqrt{9 \mu_{i}^{2}-6 \mu_{i}+9}, \\
& \left.1.5\left(\mu_{i}-1\right)-0.5 \sqrt{9 \mu_{i}^{2}-6 \mu_{i}+9},-1\right\}, i=1,2, \ldots, n
\end{aligned}
$$

Multiple numbers of -1 is $n$.

The distance spectrum of $G_{1} \square G_{2} \square G_{3}$. Lemma 6: if $\mathrm{Gi}$ (i=1, 2, 3) is connected graph,

$$
u=\left(u_{1}, u_{2}, u_{3}\right), v=\left(v_{1}, v_{2}, v_{3}\right) \in V\left(G_{1}\right) \times V\left(G_{2}\right) \times V\left(G_{3}\right)
$$

$d_{G_{1} \square G_{2} \square G_{3}}(u, v)=d_{G_{1}}\left(u_{1}, v_{1}\right)+d_{G_{2}}\left(u_{2}, v_{2}\right)+d_{G_{3}}\left(u_{3}, v_{3}\right)$

$d_{G_{1} \square G_{2} \square G_{3}}(u, v)$ represents the distance of $U, V$ in the $G_{1} \square G_{2} \square G_{3}$.

Theorem 7: let $\mathrm{Gi}(\mathrm{i}=1,2,3)$ is distance regular degree that respectively is distance regular graph of $\mathrm{Ki},|\mathrm{V}(\mathrm{Gi})=\mathrm{ni}|$, and

$$
\begin{aligned}
& \operatorname{spec}_{D}\left(G_{1}\right)=\left\{k_{1}, \mu_{2}, \mu_{3}, \ldots, \mu_{n 1}\right\} \\
& \operatorname{spec}_{D}\left(G_{2}\right)=\left\{k_{2}, \eta_{2}, \eta_{3}, \ldots, \eta_{n 1}\right\} \\
& \operatorname{spec}_{D}\left(G_{3}\right)=\left\{k_{3}, c_{2}, c_{3}, \ldots, c_{n 1}\right\}
\end{aligned}
$$

So, $\operatorname{spec}_{D}\left(G_{1} \square G_{2} \square G_{3}\right)=\left\{k_{1} n_{2} n_{3}+k_{2} n_{1} n_{3}+k_{3} n_{1} n_{2}, u_{i} n_{2} n_{3}, \eta_{j} n_{1} n_{3}, c_{t} n_{1} n_{2}, 0\right\}$,

$i=2, \ldots, n_{1}, j=2, \ldots, n_{2} ; t=2, \ldots, n_{3}$, multiple numbers of zero is $n_{1} n_{2} n_{3}-n_{1}-n_{2}-n_{3}+2$.

\section{Conclusions}

Theorem 1. If the map $G \in G_{n, \beta}$, and GG has a minimum distance spectral radius in $G_{n, \beta}$, Then there exists a non negative integers that enable $G=K_{s} \vee\left(K_{n q} \cup \overline{K_{q-1}}\right)$, among $\mathrm{q}=\mathrm{n}+\mathrm{s}-2 \beta$, $\mathrm{nq}=2 \beta-2 \mathrm{~s}+1$. This means $\mathrm{n}$ is the number of vertices, matching number is a set of connected beta graph.

Theorem 2. For an arbitrary graph $G \in G_{n, \beta}$, then

(1) If $n=2 \beta$ or $2 \beta+1$, then $\wedge_{1}(G) \geq \wedge_{1}\left(K_{n}\right)$, equality only need $G \cong K_{n}$

(2) If $\mathrm{n} \geq 2 \beta+2$, then $\wedge_{1}(G) \geq 0.5\left(-3+2 n-\beta+\sqrt{1-4 n+6 \beta+4 n^{2}-8 n \beta+5 \beta^{2}}\right)$, equality only need $G \cong K_{\beta} \vee \overline{K_{n-\beta}}$.

Theorem 3. let $\mathrm{G}$ be a connected distance regular graph, distance regular degree is $\mathrm{k}$, the corresponding distance matrix is $\mathrm{D}$, spec $(\mathrm{G}) 1=\mathrm{k}=\{\mu 1=\mathrm{k}, \mu 2, \ldots \mu \mathrm{n}\}$, and the distance between the feature graph $\mathrm{G} \odot \mathrm{K} 2$ value does not contain distance feature value of $\mathrm{G}$, then

$$
\begin{aligned}
& \operatorname{spec}_{D}\left(G \odot K_{2}\right)=\left\{-1.5+2 n+1.5 k+0.5 \sqrt{9-24 n-6 k+24 n^{2}+24 n k+9 k^{2}},\right. \\
& -1.5+2 n+1.5 k-0.5 \sqrt{9-24 n-6 k+24 n^{2}+24 n k+9 k^{2}}, 1.5\left(\mu_{i}-1\right)+0.5 \sqrt{9 \mu_{i}^{2}-6 \mu_{i}+9}, \\
& \left.1.5\left(\mu_{i}-1\right)-0.5 \sqrt{9 \mu_{i}^{2}-6 \mu_{i}+9},-1\right\}, i=2, \ldots, n
\end{aligned}
$$

Theorem 4. Let $\mathrm{Gi}(\mathrm{i}=1,2,3)$ is distance regular degree that respectively is distance regular graph of $\mathrm{Ki},|\mathrm{V}(\mathrm{Gi})=\mathrm{ni}|$, and

$$
\begin{aligned}
& \operatorname{spec}_{D}\left(G_{1}\right)=\left\{k_{1}, \mu_{2}, \mu_{3}, \ldots, \mu_{n 1}\right\} \\
& \operatorname{spec}_{D}\left(G_{2}\right)=\left\{k_{2}, \eta_{2}, \eta_{3}, \ldots, \eta_{n 1}\right\} \\
& \operatorname{spec}_{D}\left(G_{3}\right)=\left\{k_{3}, c_{2}, c_{3}, \ldots, c_{n 1}\right\}
\end{aligned}
$$

So, $\operatorname{spec}_{D}\left(G_{1} \square G_{2} \square G_{3}\right)=\left\{k_{1} n_{2} n_{3}+k_{2} n_{1} n_{3}+k_{3} n_{1} n_{2}, u_{i} n_{2} n_{3}, \eta_{j} n_{1} n_{3}, c_{t} n_{1} n_{2}, 0\right\}$, $i=2, \ldots, n_{1}, j=2, \ldots, n_{2} ; t=2, \ldots, n_{3}$, multiple numbers of zero is $n_{1} n_{2} n_{3}-n_{1}-n_{2}-n_{3}+2$ 


\section{Acknowledgement}

Shihezi University outstanding young backbone teachers training support program (the fourth batch), project number:3152LXY04032

\section{Reference}

[1] M.Q. Zhai. The structure parameters and the graph eigenvalues. East China Normal University.2010.

[2] X.L. Zhang, Laplace spectrum of a graph and distance spectrum, PhD thesis, Lanzhou University, 2009

[3] P. Li, J.S. Shi. The graph distance spectral radius of circles. Journal of East China University of Science and Technology (NATURAL SCIENCE EDITION).2010, 36 (6): 859-861.

[4] Dam E.R. van. Graphs with given diameter maximizing the spectral radius. Linear Algebra and its Applications. 2007, 426: 454-457. 